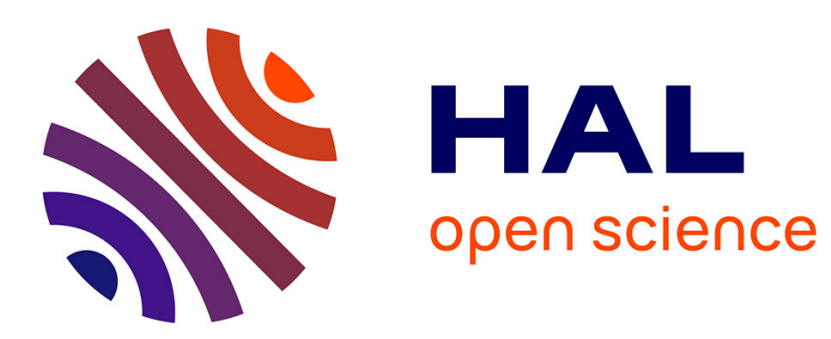

\title{
An ensemble learning framework for distributed resource allocation in inteference channels: The two user case
}

George A Ropokis

\section{To cite this version:}

George A Ropokis. An ensemble learning framework for distributed resource allocation in inteference channels: The two user case. Fourth International Balkan Conference on Communications and Networking, Sep 2021, Novi Sad, Serbia. hal-03350644

\section{HAL Id: hal-03350644 https://hal.science/hal-03350644}

Submitted on 21 Sep 2021

HAL is a multi-disciplinary open access archive for the deposit and dissemination of scientific research documents, whether they are published or not. The documents may come from teaching and research institutions in France or abroad, or from public or private research centers.
L'archive ouverte pluridisciplinaire HAL, est destinée au dépôt et à la diffusion de documents scientifiques de niveau recherche, publiés ou non, émanant des établissements d'enseignement et de recherche français ou étrangers, des laboratoires publics ou privés. 


\title{
An ensemble learning framework for distributed resource allocation in inteference channels: The two user case
}

\author{
George A. Ropokis \\ CentraleSupélec/IETR, CentraleSupélec, Campus Rennes, 35510 Cesson Sevigne, France \\ georgios.ropokis@centralesupelec.fr
}

\begin{abstract}
We focus on the problem of optimal power allocation for a two user interference channel characterized by mixed Channel State Information (CSI), which includes instantaneous information for the direct channels and statistical information for the interference channels. For this model, we introduce a general framework for optimizing the power allocation such as to maximize some generic Quality of Service (QoS) performance metric (or equivalently minimize some cost function). We model this problem as a function approximation problem where the function to be learned is the mapping between CSI and the solution to the optimization problem. We then tackle this problem borrowing ideas from ensemble learning. In particular, using generalized linear models (which are characterized by low complexity and can be implemented even at network nodes characterized by strong computational limitations), we produce different weak learners for learning to solve the considered optimization problem and based on ensemble learning theory, we combine such learners to produce stronger learners. We assess the performance of our framework by applying it on a particular resource allocation problem, and the obtained performance results indicate that the proposed approach can deliver near-optimal performance.
\end{abstract}

\section{INTRODUCTION}

While Machine Learning (ML) techniques have found several applications on the design of physical layer and resource allocation techniques for wireless communications systems, the available literature (see for example [1], [2], [3], [4], [5]) focuses on the use of advanced, computationally demanding, deep learning structures. Focusing particularly on optimal resource allocation, the seminal works in [5] and [6], propose the use of deep learning techniques following a "Learn to optimize" strategy in order to learn the association between channel conditions and optimal resource allocation (e.g., power allocation PA decisions). Given that deep learning based resource allocation algorithms proposed in references mentioned above presume the use of computational equipment capable of implementing deep learning based function approximators and classifiers on real time, its applications are limited if communication equipment characterized by very strict computational constraints is considered. Such very strict computational constraints can appear mainly in Internet of Thing (IoT) networks and fog computing environments. In such environments, the limited computational resources available to the communication/computation nodes, impose using low complexity ML based designs. Some approaches to this problem are discussed in [7] where a "Network as a Computer" architecture is introduced, where the several neurons of an Artifical Neural Network (ANN) are mapped to IoT network nodes. However, when such an approach is followed for deep learning networks, the process of calculating the output of the output layers requires an increased number of message exchanges between the IoT nodes serving as input, hidden and output layers' neurons. As a result, the computation can be influenced by unreliable communication (due to outages) and may also result to inefficient exploitation of energy resources available to power hungry IoT nodes.

An alternative to that approach is found if federated learning strategies are followed. Following such strategies, in [8], network-aware distributed learning method are proposed where devices apply local processing and send their learnt parameters to an aggregator/server. In this process, the devices have also the option of offloading their local data processing to each other. However, the work in [8], as well as in [7], considers generic ML problems and does not focus on ML assisted treatment of wireless communications related problems (such as resource allocation problems). Moreover, the system design proposed in [8], imposes the need for an aggregator/server meant to coordinate the learning and decision making process.

Deviating by the above sophisticated models, in this work we investigate the use of a distributed process for optimizing resource allocation in interference channels. We consider a two-user interference channel and discuss the problem of optimal power allocation, such as to maximize some Quality of Service (QoS) metric (e.g., weighted sum rate), or minimize some cost function (e.g., the Bit Error Rate). For this problem, we introduce a generalized ML based framework which is based on the use of simple statistical/ML tools (such as Generalized Linear Models GLMs) combined with ensemble learning techniques. In particular, in our proposed scheme, the process of learning an optimal power allocation process, is achieved by employing several low complexity "weak" learners (i.e., learners that cannot deliver by themselves highly accurate results) which calculate different approximate solutions to the optimal power allocation problem. Based on these "weak" learners, using ensemble learning based methods in order to determine/design these learners, we construct "strong" learners (i.e., learners that can deliver highly accurate results) building on the results obtained by the several "weak" learners. A significant advantage of this approach is the fact that the 
several "weak" learners are characterized by low complexity and can be implemented in a parallel fashion. As a result, the process of calculating the approximation produced by each learner can be offloaded even to equipment characterized by very limited computational resources. Moreover, the fact that the "weak" learners can be implemented in parallel allows for exploiting several of the available neighboring network nodes for computational purposes, and can reduce the number of communication hops required in order to obtain a "strong" learner. Another important advantage of our approach, is the fact that our framework does not consider a predetermined QoS metric/cost function to be used as an optimal power allocation problem. In fact, different performance optimization criteria can be defined, simply by changing the parameters of the used "weak" learners.

Finally, we also highlight that the proposed framework is based on a realistic CSI availability scenario where only the direct channels are instantaneously known for the two users, while the interference channels are only known statistically. The advantage of this CSI scenario is that it does not require some channel estimation process for determining the interference channels. As a result, it results in savings/efficiency in terms of the use of computation and energy resources, which can be important in IoT networks. Moreover, we note that in several cases, in wireless communication networks, the statistical CSI information can be inferred using location information available to the nodes. Due to these important characteristics such CSI availability scenarios where direct channel CSI is instantaneously available and interference related CSI is only statistically known have been studied in several works (see for example [9], [10]).

Based on the above, to the best of our knowledge our work is the first one to propose a generalized framework for optimal power allocation for the interference channel that can be applied for different QoS/performance optimization criteria, and builds on a realistic CSI availability scenario. Moreover, it is the first one to consider the use of ensemble learning for this purpose and can benefit from in-network parallel computation exploiting even nodes characterized by strict computational resources availability constraints.

The rest of the paper is organized as follows. In Section II, we introduce our generic system model and the considered peruser performance metrics. In Section III we present the optimal power allocation problem and some characteristic performance metrics that can be used within our framework. In Section IV we briefly discuss the ensemble learning techniques that we adopt in this paper. Following that, in Section V we discuss in detail our method for solving the optimization problem modeled by our framework. Finally, in Section VI we present simulation results which illustrate the performance of our framework for a suitable optimization problem and validate that our framework can deliver near optimal performance .

Notation: Bold lower case letters are used in order to denote vectors and bold upper case letters denote matrices. Notation $x \sim \mathcal{C N}\left(0, \sigma^{2}\right)$ denotes that random variable $x$ follows a complex circularly symmetric Gaussian distribution with zero

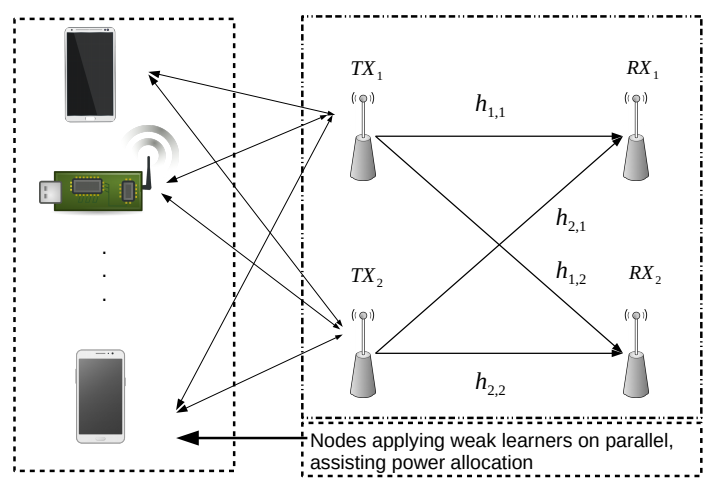

Fig. 1. The general architecture of our system model

mean and variance $\sigma^{2}$. We use notation $\mathbb{E}_{x}\{\cdot\}$ to denote the expectation operator of a random quantity over the distribution of random variable $x$. Finally we use notation $\lceil\cdot\rceil^{+}$for the operator $\max \{\cdot, 0\}$.

\section{SYSTEM MODEL}

We consider a system model such as the one in Fig. 1, where TX/RX pairs $\left\{T X_{1}, R X_{1}\right\}$ and $\left\{T X_{2}, R X_{2}\right\}$ exploit the same frequency channel for communication purposes and seek to optimize the power levels that they use. However, given strict computational resources limitations, they attempt to solve this power allocation problem in a distributed manner, by exploiting their own computational resources, as well as the computational resources of nearby network entities (e.g., neighboring users and/or network infrastructure).

In order to mathematically formulate the considered power allocation problem, we start by focusing on the signal reaching $R X_{i}, i=1,2$ and express it as as:

$$
y_{i}=h_{i, i} \sqrt{P_{i}} x_{i}+h_{j, i} \sqrt{P_{j}} x_{j}+w_{i},
$$

where $x_{i},\left(\mathbb{E}\left\{\left|x_{i}\right|^{2}\right\}=1\right)$ the data signal transmitted by $T X_{i}, i=1,2$, and $x_{j}, j \neq i$ the data signal transmitted by $T X_{j}$. Moreover, in (1), $w_{i} \sim \mathcal{C N}\left(0, \sigma^{2}\right)$ is the Additive White Gaussian Noise (AWGN) at $R X_{i}, i=1,2$, and $P_{i}$ the transmit power level used by $T X_{i}, i=1,2$. Finally, $h_{j, i}, i=$ $1,2, j=1,2$ is the channel formed between $T X_{j}$ and $R X_{i}$. Throughout our analysis, we adopt a Rayleigh fading channel model, and as a result, we assume that $h_{i, j} \sim \mathcal{C N}\left(0, \bar{g}_{i, j}\right)$. Given this signal and channel model we are now interested in optimizing power allocation $\left(P_{1}, P_{2}\right)$, such as to maximize a predetermined Quality of Service (QoS) metric. In this process, we adopt the following system design choices which determine the structure of our generic optimization framework.

\section{A. The Channel State Information availability scheme}

We assume a limited Channel State Information (CSI) scenario, where $T X_{i}, i=1,2$, has access only to direct channel state information (i.e., to coefficients $h_{1,1}, h_{2,2}$ ) and only statistical knowledge of channel coefficients $h_{2,1}, h_{1,2}$, i.e., knowledge of $\bar{g}_{1,2}=\mathbb{E}\left\{\left|h_{1,2}\right|^{2}\right\}$ and $\bar{g}_{2,1}=\mathbb{E}\left\{\left|h_{2,1}\right|^{2}\right\}$. 


\section{B. The considered performance metrics}

As our intention is to propose a generic framework able to tackle power allocation problems for a variety of Quality of service metrics, in what follows, we summarize the considered performance metrics that each one of the two users can consider.

1) User-level performance metrics: For the predetermined CSI availability scenario, we consider the following user based performance metrics.

Rate based metrics: Given the signal model in (1), one can write the instantaneous transmit rate for the $\left\{T X_{i}, R X_{i}\right\}$ transmitter-receiver pair, $i=1,2$ as:

$R_{i}\left(P_{i}, P_{j} ; g_{i, i}, g_{j, i}\right)=\log _{2}\left(1+\frac{g_{i, i} P_{i}}{g_{j, i} P_{j}+\sigma^{2}}\right)$, where $j \neq i$,

where $g_{i, i}=\left|h_{i, i}\right|^{2}$ and $g_{j, i}=\left|h_{j, i}\right|^{2}$. However, given the CSI availability limitations described earlier, calculation of $R_{i}\left(P_{i}, P_{j} ; g_{i, i}, g_{j, i}\right)$ is not possible. Instead, using knowledge of the fact that $h_{j, i}$ follows a Rayleigh channel model, and assuming knowledge of $\bar{g}_{i, j}$, one can calculate the ergodic (with respect to interference) rate as:

$$
\begin{aligned}
\mathcal{R}_{i}\left(P_{i}, P_{j} ; g_{i, i}, \bar{g}_{j, i}\right) & =\mathbb{E}_{g_{j, i}}\left\{\log _{2}\left(1+\frac{g_{i, i} P_{i}}{g_{j, i} P_{j}+\sigma^{2}}\right)\right\} \\
& =\int_{0}^{\infty} \log _{2}\left(1+\frac{g_{i, i} P_{i}}{g P_{j}+\sigma^{2}}\right) \frac{e^{-\frac{g}{g_{j, i}}}}{\bar{g}_{j, i}} d g,
\end{aligned}
$$

where $i=1,2$, and $j \neq i$. As a result, working as in [9], we can express $\mathcal{R}_{i}(\cdot, \cdot ; \cdot, \cdot)$ as:

$\mathcal{R}_{i}\left(P_{i}, P_{j} ; g_{i, i}, \bar{g}_{j, i}\right)=\frac{1}{\ln 2}\left\{U\left(\frac{g_{i} P_{i}+\sigma^{2}}{\bar{g}_{j, i} P_{i}}\right)-U\left(\frac{\sigma^{2}}{\bar{g}_{j, i} P_{j}}\right)\right\}$

where

$$
U(x)=\ln x+\exp (x) E_{1}(x),
$$

with $E_{1}(x)$ being the exponential integral [11].

User-level Bit Error Rate (BER) metrics: Besides rate based metrics, exploiting the signal model in (1) the use of Bit Error Rate (BER) based performance metrics can also be considered. For example, assuming (for the sake of simplicity) that both $T X_{1}$ and $T X_{2}$ transmit BPSK signals, focusing on the signal received by $R X_{i}$, we can see that random variable $h_{j, i} x_{j} \sqrt{P_{j}}$ is a complex circularly symmetric gaussian random variable, with variance per dimension equal to $\bar{g}_{j, i} P_{j} / 2$. Following standard approaches for determining the BER in the presence of Gaussian noise, we can then write the average (with respect to $\left.g_{j, i}\right)$ BER as:

$$
P_{e}\left(P_{1}, P_{2} ; g_{i, i}, \bar{g}_{j, i}\right)=0.5 \operatorname{erfc}\left(\sqrt{\frac{g_{i, i} P_{i}}{\bar{g}_{j, i}+2 \sigma^{2}}}\right),
$$

with $\operatorname{erfc}(\cdot)$ being the complementary error function.

Given the above performance metrics, in the following section we present in more detail the considered optimization problem and our approach for solving it.

\section{THE CONSIDERED OPTIMIZATION PROBLEM}

Exploiting the performance metrics described in Section II, we consider the problem of minimizing a generic cost function which involves the abovementioned performance metrics. For this purpose, in this work we start by considering only simple box constraints, for our optimization problem. As a result, we can write the optimal power allocation problem in the form:

$$
\begin{aligned}
& \text { minimize: } C\left(P_{1}, P_{2} ; g_{1,1}, g_{2,2}, \bar{g}_{1,2}, \bar{g}_{2,1}\right) \\
& \text { subject to: } 0 \leq P_{1} \leq P_{\max }, \quad 0 \leq P_{2} \leq P_{\max },
\end{aligned}
$$

where $P_{\max }$ is the maximum allowable power level per user, and $C\left(P_{1}, P_{2} ; g_{1,1}, g_{2,2}, \bar{g}_{1,2}, \bar{g}_{2,1}\right)$ the considered generic performance metric. In what follows, For the sake of illustration, we present some typical problems that can be seen as special case of this problem.

Weighted sum rate maximization: As we know the Weighted Sum Rate (WSR) can be expressed as:

$$
S=\alpha_{1} R_{1}\left(P_{1}, P_{2} ; g_{1,1}, g_{2,1}\right)+\alpha_{2} R_{2}\left(P_{1}, P_{2} ; g_{2,2}, g_{2,1}\right) \text {. }
$$

where $\alpha_{1}, \alpha_{2}$ non-negative weights satisfying the constraint $\alpha_{1}+\alpha_{2}=1$. Building on the considered CSI availability scenario, we can then define an analogous to the WSR as the ergodic (with respect to interference) WSR, as:

$$
\mathcal{S}=\alpha_{1} \mathcal{R}_{1}\left\{P_{1}, P_{2} ; g_{1,1}, \bar{g}_{2,1}\right\}+\alpha_{2} \mathcal{R}_{2}\left\{P_{1}, P_{2} ; g_{2,2}, \bar{g}_{1,2}\right\} .
$$

Exploiting this definition of $\mathcal{S}$, one can write the optimal power allocation, with respect to the WSR, for the considered CSI availabilty scenario as:

$$
\begin{aligned}
& \text { minimize: }-\mathcal{S} \\
& \text { subject to: } 0 \leq P_{1} \leq P_{\max }, \quad 0 \leq P_{2} \leq P_{\max } .
\end{aligned}
$$

Max-min rate based optimization: As an alterantive to WSR optimization, several works on optimal power allocation focus on introducing fairness in the power allocation process by considering max-min optimization criteria. In case that such an approach is followed for our proposed CSI availability scenario, one can consider introducing fairness by introducing the following optimization problem:

maximize: $\min \left\{\mathcal{R}_{1}\left(P_{1}, P_{2} ; g_{1,1}, \bar{g}_{2,1}\right), \mathcal{R}_{2}\left(P_{1}, P_{2} ; g_{2,2}, \bar{g}_{1,2}\right)\right\}$ subject to: $0 \leq P_{1} \leq P_{\max }, 0 \leq P_{2} \leq P_{\max }$.

Reliability and fairness based optimization: Focusing again on fairness based designs and on reliability based performance metrics (such as the BER) one can consider the following problem:

minimize: $\max \left\{P_{e}\left(P_{1}, P_{2} ; g_{1,1}, \bar{g}_{2,1}\right), P_{e}\left(P_{1}, P_{2} ; g_{2,2}, \bar{g}_{1,2}\right)\right\}$ subject to: $0 \leq P_{1} \leq P_{\max }, 0 \leq P_{2} \leq P_{\max }$,

as one more instance of the generic optimal power allocation problem ( $\mathrm{P} 1)$

By inspecting optimization problems (P2), (P3) and (P4) we can easily observe that obtaining the solution to these 
problems in closed form (by applying for example KKT conditions) becomes complicated and requires solving systems of non linear equations. Moreover, applying, as an alternative, iterative optimization techniques may also lead to increased implementation complexity mainly due to the need of calculating the cost function value at multiple points, as well as due to the need for calculating the gradient vector of the cost function, for every point visited by the iterative algorithm (in case of gradient based iterative algorithms). Therefore, taking into account the abovementioned complexity requirements of standard optimization approaches for tackling some standard problems of the form (P1), in our work we follow a different strategy that seeks to approximate the solution to optimization problems of the form (P1), by exploiting simple statistical based approximations, i.e., GLM based approximations to the optimal power allocation. As it will become evident in the numerical evaluation section, using multiple GLMs of this type and distributing the problem of calculating the resulting approximations to nodes of our network, we can exploit the intelligence of each one of the network nodes, and reach satisfactory power allocation decisions even in cases where the several collaborating nodes are characterized by strict complexity limitations. When using such an approach, the process of solving problem (P1), then boils down to appropriately selecting the strategy for training/determining the GLM model to be applied at each one of the network nodes participating in the optimization process. For this process, we propose to use techniques based on ensemble learning, and more particularly, boosting. The basic principles of both ensemble learning and boosting are briefly described in what follows.

\section{ENSEMBLE LEARNING}

The term ensemble learning refers to the set of machine learning techniques that are produced by training multiple weak learners, i.e., learners characterized by a low level of accuracy, in order to solve a machine learning problem and then combining them. While under this broad category of methods several subcategories can be found, for our purposes we will only consider methods based on applying different statistical models/weak learners to the same training set as well as boosting methods, i.e., methods where several weak learners are trained in a manner such that a learner focuses on producing a model having a good accuracy performance for the training samples for which all previously trained learners have been found to perform poorly [12].

\section{OUR PROPOSED METHOD}

As described in Section II and Fig. 1, in our considered optimization approach, nodes $T X_{1}$ and $T X_{2}$ attempt to solve a problem of the form (P1) by exploiting their own computational resources, as well as the computational resources of a set of "helper" nodes, denoted as $\mathcal{H}=\left\{H_{1}, \ldots, H_{K}\right\}$. Assuming a strict constraint on the available computational resources (such as the constraints that correspond to the computational resources of IoT nodes) we assume that nodes $T X_{1}$ and $T X_{2}$ and every node in $\mathcal{H}$ uses a different, low complexity weak learner for the considered optimization problem. However, by using ensemble learning (i.e., boosting) techniques in order to properly design these weak learners, one can create strong learners able to provide highly accurate approximate solutions to the considered optimization problems. In order to further explain this process, in what follows, we first describe the weak learning approaches considered in our work.

\section{A. The considered "weak" learners}

Aiming at satisfying strict constraints concerning the available computational resources, we propose the use of GLMs [13] and briefly describe them in what follows.

1) Generalized linear models: When a GLM is used, given a set of independent observations $Y_{1}, \ldots, Y_{N}$, along with corresponding vectors of explanatory variables $\mathbf{x}_{1}, \ldots, \mathbf{x}_{N}$, where $\mathbb{E}\left\{Y_{i}\right\}=\mu_{i}$, we assume that for a given monotone, differentiable function $g(\cdot)$ (the so-called link function in the GLM terminology) it holds that:

$$
g\left(\mu_{i}\right)=\mathbf{x}_{i}^{T} \mathbf{b},
$$

where $\mathbf{b}$ a predictor vector. As a result, the construction of a GLM requires producing an estimate $\hat{\mathbf{b}}$ for $\mathbf{b}$ in (9), given $g(\cdot)$, observations $Y_{1}, \ldots, Y_{N}$ and their corresponding vectors of explanatory variables $\mathbf{x}_{1}, \ldots, \mathbf{x}_{N}$. A solution to this estimation problem can then be found using a Maximum Likelihood (ML) estimator, following the process described in [13].

Inversely, given a predictor vector $\mathbf{b}$ obtained after a GLM training process and a vector of explanatory variables $\mathbf{x}$, we can estimate $y$ corresponding to this categorical vector as:

$$
\hat{y}=g^{-1}\left(\mathbf{x}_{i}^{T} \mathbf{b}\right) .
$$

2) Learning power allocation decisions using GLMs: Based on the previous description of GLMs, we propose using GLMs for learning the association between the power allocation that user $i, i=1,2$, should employ and a vector of explanatory variables depending on values $g_{1,1}, g_{2,2}$, for the given average fading conditions $\bar{g}_{i, j}, i=1,2, j=1,2$. Following such an approach, and aiming to capture also the effect of channel imbalances on the power allocation, we propose to learn the power allocation decisions for $T X_{1}$ and $T X_{2}$, by training two different GLMs, one corresponding to the power allocation decision for the TX characterized by the highest channel gain, i.e., $T X_{i}$ with $i$ such that $g_{i, i}=g_{\max }=\max \left\{g_{1,1}, g_{2,2}\right\}$ and one corresponding to the power allocation decision for the TX characterized by the smallest channel gain i.e. $T X_{j}$ with $j$ such that $g_{j, i}=g_{\min }=$ $\min \left\{g_{1,1}, g_{2,2}\right\}$.

Let us now focus on the design of the GLM used to model the power allocation decision of $T X_{i}$, with $i$ such that $g_{i, i}=$ $g_{\max }$. Let $\tilde{P}$ be the power allocation decision for this $T X$. In order to model the association between random variable $\tilde{P}$ and the GLM we propose to use as candidate link functions the logit link function, defined as [13]:

$$
\operatorname{logit}(\mu)=\log \left(\frac{\mu}{1-\mu}\right), \quad \mu \in(0,1)
$$


and the probit link function, defined as [13]:

$$
\operatorname{probit}(\mu)=\Phi^{-1}(\mu), \mu \in(0,1) \text {. }
$$

While in the statistical literature these two functions are mostly used in order to estimate GLMs for binary, categorical observations, the fact that they limit the considered values for $\mu$ in the interval $(0,1)$ is coherent with the box constraints of our problem. In more detail given the constraints $0 \leq P_{1} \leq P_{\max }$ and $0 \leq P_{2} \leq P_{\max }$ if we introduce the variable:

$$
Y=\frac{\tilde{P}+\varepsilon}{P_{\max }+c \varepsilon}
$$

with $0<\varepsilon<<P_{\max }$ and $c \rightarrow 1$, with $c>1$, we obtain that the value $\mu_{i}=\mathbb{E}\left\{Y_{i}\right\}$ will belong in the interval $(0,1)$. Therefore this choice for the candidate link functions is validated. The ML predictor vector $\mathbf{b}$ can then be obtained using the process described in [13, Chapter 4]. Following a similar approach, the power allocation for the user $T X_{j}$ where $j$ is such that $g_{j, j}=\min \left\{g_{1,1}, g_{2,2}\right\}$ can also be constructed. Further details in this process are ommited due to space limitations.

Before proceeding with describing next steps of our proposed method, we comment that while following the above approach for learning power allocation decisions requires changing the applied predictors every time that that average statistics change, in case of low mobility, we can assume that the channel statistics remain constant for sufficiently long time intervals, reducing the need for frequent updates of the predictors. Moreover, we comment that for practical purposes, the training process for the GLMs, i.e., the calculation of the predictor vectors can be performed offline for representative average fading conditions and become accessible to users on demand through network infrastructure and edge intelligence.

\section{B. Building "strong” predictors by combining different GLMs}

As explained earlier, a straight forward way for creating different learners is by considering different statistical models for the connection between observations $Y_{1}, \ldots, Y_{N}$ and the vector of explanatory variables. Restricting ourselves to GLMs, this can be ensured by using different link functions to train different GLMs for the same training dataset. As a result, with this in mind, we propose employing a number of at least two GLM based predictors, with at least one corresponding to a logit link function and one corresponding to a probit function. Additional predictors can then be derived by exploiting the concepts of boosting. Further details for constructing additional GLM based predictors are given in the numerical evaluation section.

\section{The final power allocation learning framework}

Having described our GLM based prediction schemes, we can now summarize our power allocation prediction framework as the sequence of the following three steps:

1) Forwarding CSI to all involved nodes: Clearly, the first step of the algorithm requires the exchange of all CSI between $T X_{1}$ and $T X_{2}$. Assuming a broadcast transmission, at the end of this step, values $g_{1,1}, g_{2,2}$ and $\bar{g}_{1,2}, \bar{g}_{2,1}$ become available to $T X_{1}$ and $T X_{2}$ as well as to all nodes in $\mathcal{H}$.

2) Calculating candidate power allocations: During this step, each one of the nodes $T X_{1}$ and $T X_{2}$ calculates a number of candidate optimal power allocations as well as the corresponding value for the selected cost function in (P1). The same process (using however different GLM estimators) is also applied as each one of the "helper nodes" in $\mathcal{H}$.

3) Selecting the final power allocation As a final step, $T X_{1}$ forwards to $T X_{2}$ its best candidate power allocation (and the corresponding cost function value) and similarly $T X_{2}$ forwards to $T X_{1}$ its best candidate allocation (and the corresponding cost function value). Each one of the "helpers" also forwards its best candidate power allocation to both $T X_{1}, T X_{2}$, along with the corresponding cost function values. Let $\mathcal{P}$ be the set of candidate power values available at both $T X_{1}$ and $T X_{2}$ at the end of this process, and $\left(\hat{P}_{1}, \hat{P}_{2}\right) \in \mathcal{P}$ the allocation which results in the smallest cost function value. We then select this power allocation as the one to be used for transmission.

\section{NUMERICAL EVALUATION AND DISCUSSION}

We now consider applying our proposed framework for a power allocation problems of the form (P1). To this end, we consider a symmetric placement of the $\left\{T X_{i}, R X_{i}\right\}$ pairs. In more detail, we set $T X_{1}$ to be the origin of our coordinate system and place $T X_{2}$ at coordinates $\left(0, d_{t}\right)$ where $d_{t}$ the distance between the two transmitters expressed in meters. Furthermore, we place $R X_{1}$ at coordinates $\left(d_{r}, 0\right)$, i.e., at a distance $d_{r}$ from $T X_{1}$, and $R X_{2}$ at coordinates $\left(d_{r}, d_{t}\right)$, i.e., at a distance $d_{r}$ from $R X_{2}$. Following such an approach, and using an inverse power pathloss model, $\bar{g}_{i, j}$ is given as:

$$
\bar{g}_{i, j}=\frac{\mathcal{A}}{\left(\operatorname{dist}\left(T X_{i}, R X_{j}\right) / d_{0}\right)^{p}}
$$

where $\operatorname{dist}\left(T X_{i}, R X_{j}\right)$ the distance between $T X_{i}$ and $R X_{j}$, $p$ the pathloss exponent, and $\mathcal{A}$ the nominal power attenuation at a reference distance $d_{0}$. As a result of this placement, we obtain that $\bar{g}_{1,1}=\bar{g}_{2,2}$ and $\bar{g}_{1,2}=\bar{g}_{2,1}$. Concerning the exact values of the pathloss parameters, we follow the channel model used in [14] and set $d_{0}=1$ and $\mathcal{A}=-30[d B]$. Moreover, we set the pathloss exponent equal to $p=2.5$. Finally, as in [14], we set the noise variance to be equal to $-90 \mathrm{~d} B$. For this fading scenario, we consider the following optimization problem that fall under the umbrella of our framework.

\section{A. BER optimal power allocation}

We consider a problem of the form (P4). For this problem, after setting $d_{r}=d_{t}=10$ meters we have considered generating $10^{3}$ channel realizations following the Rayleigh fading assumption discussed earlier. Using these channel realizations, we have designed separate GLMs for learning the power allocation of the user characterized by the strongest channel gain and the user characterized by the weakest channel gain. 
For a given channel realization $\left(g_{1,1}, g_{2,2}\right)$ we have solved the optimal power allocation by using function fmincon of MATLAB $^{\mathrm{TM}}$ with multiple different initializations such as to avoid as much as possible the event of being trapped at a local optimum. For a given channel realization $\left(g_{1,1}, g_{2,2}\right)$, for the GLM for learning the optimal power allocation for the $\left\{T X_{i}, R X_{i}\right\}$ pair corresponding to the strongest direct channel we have considered as observed variables $Y_{k}, k=1, \ldots, 10^{3}$, the normalized power allocation values obtained by applying the process described in (13). Moreover, for any channel realization $\left(g_{1}, g_{2}\right)$ we defined the explanatory variables as:

$$
\mathbf{x}=\left[1, g_{1,1}, g_{2,2}, g_{1,1}^{2}, g_{1,2}^{2}, g_{1,1} g_{2,2}\right]^{T} .
$$

Concerning the GLMs which we considered, we trained four different GLM schemes with the first two obtained by using a logit link function and the two remaining using a probit link function. Starting from the logit based GLMs, the first one was trained using the full training dataset. Following that, for each one of the training examples, the absolute error between the BER obtained by solving (P4) with fmincon and the BER obtained by applying the learned power allocation was calculated. By normalizing this absolute error by the optimal BER obtained by fmincon, the performance degradation due to imperfect learning was evaluated for each one of the training examples. Following that, using a boosting principle, the $25 \%$ of the inital training dataset which corresponded to the worse performance degradation was used in order to train a second logit based GLM model. Using the same process the GLMs for the probit link function were also constructed. Finally in a similar fashion, the GLMs for learning the power allocation for the weaker direct channel were also constructed.

For the purposes of assessing the performance of learned power allocation, we then considered applying the learned power allocation scheme for a test dataset of $10^{3}$ examples. For each one of these examples, we calculated the cost function value of (P4) for the learned power allocation as well as the optimal cost function value obtained by solving (P4) using fmincon. Let $B \hat{E} R_{k}$ be the cost function value obtained by applying the learning based power allocation for the $k$-th test example and $B E R_{k}^{\star}$ the cost function value obtained when the optimal power allocation is applied. Using these quantities, we can express the relative error (with respect to the optimal cost function value for the $k$-th test sample) as:

$$
r_{k}=\frac{B \hat{E} R_{k}-B E R_{k}^{\star}}{B E R_{k}^{\star}}, k=1, \ldots, 1000 .
$$

In Fig. 2 we plot the histogram of $r_{k}$. As we can see from the plotted results, for the vast majority of channel realizations, the relative error between the cost function value obtained at the learned power allocation and the value obtained at the optimal power allocation is negligible. In fact for nearly $95 \%$ of channel realizations, this error is less than 0.05 . As a result for the vast majority of cases, our approach results in power allocations characterized by very small suboptimality gap.

Results of the same quality of approximation were also obtained for other problems of the form (P1). The results are

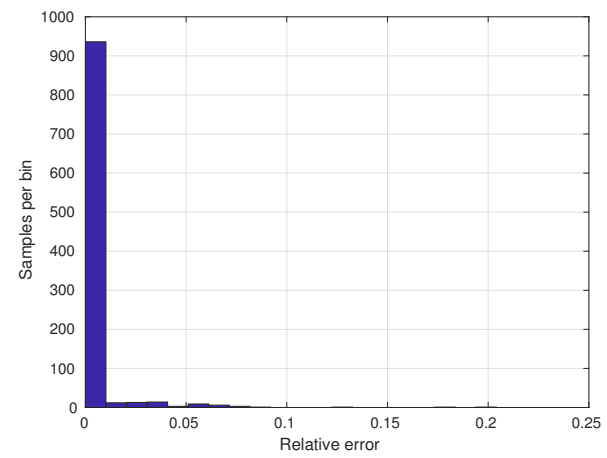

Fig. 2. The histogram of the relative error of the cost function value (with respect to the optimal cost function value) when the learn power allocation is applied in (P4).

not presented due to space limitations. Finally, we set as future objective the extension of our model to resource allocation problems with more complicated constraints and more users.

\section{REFERENCES}

[1] L. Dai, R. Jiao, F. Adachi, H. V. Poor, and L. Hanzo, "Deep learning for wireless communications: An emerging interdisciplinary paradigm," IEEE Wireless Communications, vol. 27, no. 4, pp. 133-139, 2020.

[2] T. O'Shea and J. Hoydis, "An introduction to deep learning for the physical layer," IEEE Transactions on Cognitive Communications and Networking, vol. 3, no. 4, pp. 563-575, 2017.

[3] Z. Qin, H. Ye, G. Y. Li, and B.-H. F. Juang, "Deep learning in physical layer communications," IEEE Wireless Communications, vol. 26, no. 2, pp. 93-99, 2019.

[4] L. Liang, H. Ye, G. Yu, and G. Y. Li, "Deep-learning-based wireless resource allocation with application to vehicular networks," Proceedings of the IEEE, vol. 108, no. 2, pp. 341-356, 2020.

[5] H. Sun, X. Chen, Q. Shi, M. Hong, X. Fu, and N. D. Sidiropoulos, "Learning to optimize: Training deep neural networks for interference management," IEEE Transactions on Signal Processing, vol. 66, no. 20, pp. 5438-5453, 2018.

[6] M. Eisen, C. Zhang, L. F. O. Chamon, D. D. Lee, and A. Ribeiro, "Learning optimal resource allocations in wireless systems," IEEE Transactions on Signal Processing, vol. 67, no. 10, pp. 2775-2790, 2019.

[7] E. Di Pascale, I. Macaluso, A. Nag, M. Kelly, and L. Doyle, "The network as a computer: A framework for distributed computing over iot mesh networks," IEEE Internet of Things Journal, vol. 5, no. 3, pp. 2107-2119, 2018.

[8] Y. Tu, Y. Ruan, S. Wagle, C. G. Brinton, and C. Joe-Wong, "Networkaware optimization of distributed learning for fog computing," in IEEE INFOCOM 2020 - IEEE Conference on Computer Communications, 2020, pp. 2509-2518.

[9] G. A. Ropokis, D. Gesbert, and K. Berberidis, "Rate optimal power policies in underlay cognitive radios with limited channel feedback," in 2013 IEEE 24th Annual International Symposium on Personal, Indoor, and Mobile Radio Communications (PIMRC), 2013, pp. 740-744.

[10] M. C. Filippou, G. A. Ropokis, D. Gesbert, and T. Ratnarajah, "Joint sensing and reception design of simo hybrid cognitive radio systems," IEEE Transactions on Wireless Communications, vol. 15, no. 9, pp. 6327-6341, 2016.

[11] M. Abramowitz and I. A. Stegun, Handbook of Mathematical Functions with Formulas, Graphs, and Mathematical Tables, 9th ed. New York City: Dover, 1964.

[12] R. E. Schapire and Y. Freund, Boosting: Foundations and Algorithms. The MIT Press, 2012.

[13] A. J. Dobson, An introduction to generalized linear models, 2nd ed. Chapman \& Hall/CRC Boca Raton, 2002.

[14] G. A. Ropokis, "Multi-relay cooperation with self-energy recycling and power consumption considerations," in 2019 International Conference on Wireless and Mobile Computing, Networking and Communications (WiMob), 2019, pp. 268-275. 